\title{
La cirugía de catarata disminuye la tasa de accidentes automovilís- ticos en personas mayores
}

Impact of Cataract Surgery on Motor Vehicle Crash Involvement by Older Adults. Owsley C, McGwin G, Sloane M y col. JAMA 2002; 288:841849.

\begin{abstract}
Objetivo
Determinar el impacto de la cirugía de catarata en el riesgo de padecer accidentes automovilísticos en adultos mayores, dentro de los 4 a 6 años posteriores a la cirugía, comparando con adultos mayores que presentan catarata, pero no eligen la cirugía.
\end{abstract}

\section{Diseño}

Estudio de cohorte prospectivo.

\section{Lugar}

Base de datos de 277 pacientes en clínicas de Birminghan, Alabama, EE.UU.

\section{Pacientes}

Se establecieron dos grupos de pacientes que cumplieran ciertos criterios: catarata en 1 o en 2 ojos con agudeza visual con corrección de 20/40 o menos, sin cirugía de catarata previa en algún ojo, cirugía de catarata previamente recomendada por un oftalmólogo debido a problemas visuales del paciente, pacientes con seguro médico, edad mayor o igual a 55 años, vivir independientemente en la comunidad, que presenten licencia de conducir en Alabama y que la utilicen. Un grupo de pacientes $(n=174)$ eligió la cirugía de catarata mientras que el otro grupo $(n=103)$ no la eligió como tratamiento para sus problemas de visión.

\section{Evaluación de factores pronósticos}

Se evaluaron 3 parámetros de función visual de cada ojo por separado: agudeza visual, sensibilidad al contraste y deslumbramiento.También fueron estudiados el estado cognitivo, velocidad y habilidad de atención, depresión, estado de salud general, por ser considerados parámetros de confusión y estar asociados a accidentes automovilísticos.

\section{Medición del resultado principal}

Se identificaron estadísticas descriptivas para factores demográficos, médicos, de función visual e índice de accidentes comparando entre los dos grupos de pacientes.La variable principal fue la tasa de accidentes por millas de viaje.

\section{Resultados principales}

Comparando entre los dos grupos, no hubo diferencias en relación a edad, años de educación y millas anuales. No hubo diferencias significativas con respecto a las condiciones médicas de los dos grupos. El $13 \%$ de los pacientes que eligieron la cirugía y el $25 \%$ de los que no la eligieron tenían patología ocular asociada.

La agudeza visual y la sensibilidad al contraste antes de la cirugía fue peor en los dos ojos de los pacientes que eligieron la opción quirúrgica mientras que el deslumbramiento basal fue más acentuado en el grupo que eligió la opción no quirúrgica. No hubo diferencias entre los grupos en la tasa de accidentes en los 5 años previos al estudio.

El riesgo relativo (RR) no ajustado comparando el grupo con cirugía en relación al grupo sin cirugía fue de $0,64 \%(0,37$ a 1,13$)$. Ajustándolo según raza, agudeza visual y sensibilidad al contraste el $R R$ fue de 0,47 (IC95\% 0,23 a 0,94).

La tasa absoluta de reducción de accidentes asociada al grupo de cirugía de catarata fue 4,74 accidentes por millón de millas de viaje, partiendo de una base de 8,95 accidentes por millón de millas de viaje (la tasa de accidentes entre el grupo de no cirugía).

\section{Conclusiones}

Pacientes con catarata que eligieron la opción quirúrgica tuvieron la mitad de la tasa de accidentes automovilísticos durante el período de seguimiento comparando con el grupo que no eligió la cirugía. Por lo tanto, se puede considerar el beneficio secundario de la cirugía de catarata para la seguridad de conductores automovilísticos mayores, reduciendo el índice de accidentes.

\section{Comentario}

En los cinco años previos al desarrollo del estudio, la tasa de accidentes para los dos grupos fueron similares. Durante el seguimiento, los pacientes que eligieron la opción quirúrgica aumentaron la tasa un $27 \%$ en comparación con el otro grupo que aumentó un $75 \%$.Un hecho a considerar es que el grupo sin cirugía maneja más de noche que el grupo con cirugía.

Dentro de los parámetros estudiados entre los grupos sólo 3 (agudeza visual, sensibilidad al contraste y raza) fueron consideradas como potenciales factores de confusión y por ello fueron incluidos en el estudio ajustado ${ }^{1,2}$.

El diseño aleatorizado en un trabajo de investigación clínica representa la mejor evidencia científica, emplear este tipo de diseño para evaluar la relación entre cirugía de catarata y el riesgo de accidentes no es factible debido a que la cirugía se considera una medida estándar de cuidado. Por lo tanto, la elec- ción de esta metodología en el presente estudio (estudio de cohorte prospectivo) representa la mejor opción posible ${ }^{2}$.

Otro ítem a considerar para disminuir el riesgo de accidentes en mayores es tratar las patologías asociadas que pueden ocasionar alteraciones visuales ${ }^{3,4}$.

Conclusión de los comentadores: Se ha demostrado que la mejora de la función visual que la cirugía de catarata ocasiona es un instrumento para disminuir la tasa de accidentes automovilísticos. Sin embargo, la misma es una intervención quirúrgica y como tal no está libre de complicaciones. La decisión quirúrgica debería depender de la realización de un análisis minucioso de los riesgos y beneficios que la cirugía ocasiona teniendo en cuenta las necesidades de cada paciente y del estilo de vida que mantiene ${ }^{5}$.

Dr. Eduardo Mayorga Argañaraz - Dra. Carolina M. Gentile [ Servicio de Oftalmología - Hospital Italiano de Buenos Aires ]

\section{Referencias}

1. National Highway Traffic Safety Administration. Addressing the Safety Issues Related to Younger and Older Drivers.A report to Congress.Washington, DC:US Dept of Transportation; January 19,1993

2. National Highway Traffic Safety Administration.Conference on Research and Development Needed to improve Safety and Mobility of older drivers. Washington, DC:US Dept of Transportation; 1989 Report No DOT HS 807316.

3. Kahn HA, Leibowitz HM, Ganley JP, et al.The Framingham Eye Study. I. Outline and mayor prevalence findings. Am J Epidemiol. 1977:106:17-41.

4. West SK, Munóz B, Schein OD, et al Racial differences in lens opacities the Salisbury Eye Evaluation (SEE) Proyect Am J Epidemiol 1998; 148:1033-1039.

5. Owsley C, Stalvey BT, Wells J, Soane ME, Mc Gwin G Jr.Visual risk factors for crash involvement in older drivers with cataract. Arch Ophtalmol. 2001:119:881-887. 